\title{
Efeito do treinamento resistido em mulheres com fibromialgia: Um estudo de revisão
}

\author{
Effect of resistance training in women with fibromyalgia: A review study \\ Efecto del entrenamiento de resistencia en mujeres con fibromialgia: Un estudio de revisión
}

Recebido: 05/04/2021 | Revisado: 21/04/2021 | Aceito: 22/04/2021 | Publicado: 03/05/2021

Felipe Junio Barbosa

ORCID: https://orcid.org/0000-0002-1914-9310 Universidade do Estado de Minas Gerais, Brasil E-mail: felipebarbosa23@hotmail.com

Jaqueline dos Reis Moreira Lacerda ORCID: https://orcid.org/0000-0002-0227-553X Universidade do Estado de Minas Gerais, Brasil E-mail: jaquelinerml15@gmail.com

Gislaine Cristina-Souza ORCID: https://orcid.org/0000-0001-7770-4514 Universidade do Estado de Minas Gerais, Brasil E-mail: gislaine.souza@uemg.br

Brandel José Pacheco Lopes Filho ORCID: https://orcid.org/0000-0001-6666-9637 Universidade do Estado de Minas Gerais, Brasil E-mail: brandelfilho@gmail.com

Bruno de Freitas Camilo

ORCID: https://orcid.org/0000-0002-3039-2194 Universidade do Estado de Minas Gerais, Brasil

E-mail: brunodefreitascamilo@yahoo.com.br

\begin{abstract}
Resumo
A fibromialgia é uma síndrome reumática que causa dor musculoesquelética crônica disseminada, por um período maior que três meses, cujos sintomas são dores nos tecidos moles como músculos, tendões ou ligamentos. O objetivo desta revisão foi analisar estudos que avaliaram a influência do treinamento resistido sobre a saúde de mulheres com fibromialgia. Trata-se de uma revisão integrativa de estudos publicados entre 2016 e 2020. A busca e seleção dos estudos foi realizada a partir dos termos: fibromyalgia OR fibromyalgia syndrome AND women AND adults AND resistance training. As bases de dados utilizadas na busca foram Pubmed, SciELO, Biblioteca Virtual em Saúde (BVS). Inicialmente, foram identificados 1.368 artigos. Após a aplicação do filtro (estudos publicados nos últimos 5 anos), 838 artigos apresentaram relevância, sendo que 604 estavam indexados no Pubmed e 234 na BVS. Destes, 813 artigos foram excluídos após a leitura de títulos e resumos e 25 foram selecionados para leitura na íntegra. Desse total, 14 artigos foram excluídos com justificativa e 11 atenderam aos critérios de inclusão. Constatou-se que o treinamento resistido é um importante método não medicamentoso capaz de atenuar os efeitos da fibromialgia em mulheres adultas, contribuindo para a melhoria da qualidade de vida, saúde física e mental. Deste modo, o treinamento resistido contribuiu para a redução da dor e fadiga, melhora do condicionamento físico, aumento da força e resistência, redução da depressão, melhora do humor e da qualidade de vida nos domínios relacionados aos aspectos físicos, sociais, emocionais, mentais e à vitalidade.
\end{abstract}

Palavras-chave: Treinamento de resistência; Exercício físico; Mulher; Saúde do adulto; Dor.

\begin{abstract}
Fibromyalgia is a rheumatic syndrome that causes widespread chronic musculoskeletal pain for more than three months, the symptoms of which are pain in soft tissues such as muscles, tendons or ligaments. The aim of this review was to analyze studies that assessed the influence of resistance training on the health of women with fibromyalgia. This is an integrative review of studies published between 2016 and 2020. The search and selection of studies was carried out using the terms: fibromyalgia OR fibromyalgia syndrome AND women AND adults AND resistance training. The databases used in the search were Pubmed, SciELO, Virtual Health Library (VHL). Initially, 1.368 articles were identified. After applying the filter (studies published in the last 5 years), 838 articles were relevant, of which 604 were indexed in Pubmed and 234 in the VHL. Of these, 813 articles were excluded after reading titles and abstracts and 25 were selected for reading in full. Of this total, 14 articles were excluded with justification and 11 met the inclusion criteria. Resistance training was found to be an important non-drug method capable of mitigating the effects of fibromyalgia in adult women, contributing to the improvement of quality of life, physical and mental health. In this way, resistance training contributed to reducing pain and fatigue, improving physical conditioning, increasing strength and endurance, reducing depression, improving mood and quality of life in the domains related to physical, social, emotional, mental aspects and vitality.
\end{abstract}


Keywords: Resistance training; Exercise; Women; Adult health; Pain.

\section{Resumen}

La fibromialgia es un síndrome reumático que causa dolor musculoesquelético crónico generalizado durante más de tres meses, cuyos síntomas son dolor en tejidos blandos como músculos, tendones o ligamentos. El objetivo de esta revisión fue analizar los estudios que evaluaron la influencia del entrenamiento de resistencia en la salud de las mujeres con fibromialgia. Es una revisión integradora de estudios publicados entre 2016 y 2020. La búsqueda y selección de estudios se realizó utilizando los términos: fibromyalgia OR fibromyalgia syndrome AND women AND adults AND resistance training. Las bases de datos utilizadas en la búsqueda fueron Pubmed, SciELO, Biblioteca Virtual en Salud (BVS). Inicialmente, se identificaron 1.368 artículos. Después de aplicar el filtro (estudios publicados en los últimos 5 años), 838 artículos fueron relevantes, de los cuales 604 fueron indexados en Pubmed y 234 en la BVS. De estos, 813 artículos fueron excluidos después de leer títulos y resúmenes y 25 fueron seleccionados para su lectura completa. De este total, 14 artículos fueron excluidos con justificación y 11 cumplieron los criterios de inclusión. Se descubrió que el entrenamiento de resistencia es un importante método no farmacológico capaz de mitigar los efectos de la fibromialgia en mujeres adultas, contribuyendo a mejorar la calidad de vida, la salud física y mental. De esta manera, el entrenamiento de resistencia contribuyó a la reducción del dolor y fatiga, mejora del acondicionamiento físico, aumento de la fuerza y la resistencia, reducción de la depresión, mejora del estado de ánimo y la calidad de vida en dominios relacionados con aspectos físicos, sociales, emocionales, mentales y de vitalidad.

Palabras clave: Entrenamiento de resistencia; Ejercicio físico; Mujer; Salud del adulto; Dolor.

\section{Introdução}

A fibromialgia é uma síndrome reumática que causa dor musculoesquelética crônica disseminada e acomete os lados direito e esquerdo do corpo, abaixo e acima da cintura, por um período maior que 3 meses, cujos sintomas são dores nos tecidos moles como músculos, tendões ou ligamentos (Wolfe et al., 2016). A fadiga configura-se como outro sintoma típico da fibromialgia e pode desencadear perda de memória, má qualidade do sono (Clauw, 2014), irritabilidade, problemas de humor, déficit de atenção (Sluka \& Clauw, 2016) e distúrbios psicológicos como depressão e ansiedade, afetando diretamente a qualidade de vida e o desempenho em funções sociais e ocupacionais dos indivíduos (Andrade, de Azevedo Klumb Steffens, Peyré Tartaruga, Sieczkowska, \& Torres Vilarino, 2018). Por ser uma doença complexa, a fisiopatologia da fibromialgia não é completamente clara, dificultando o seu diagnóstico (Häuser et al., 2015).

Alguns estudos investigaram métodos alternativos que poderiam atenuar os sintomas da fibromialgia, tais como terapia cognitivo-comportamental e exercícios físicos (Bidonde et al., 2017; Okifuji \& Turk, 2015). A prática de exercício físico é caracterizada como uma estratégia não medicamentosa para o tratamento da fibromialgia (Lima, Badaró, \& Ladeia, 2019), sendo seus benefícios observáveis, inclusive, a curto prazo (Jorge, Tomikawa, \& Jucá, 2007). Além disso, a prática de exercícios de fortalecimento tem sido altamente recomendada para o tratamento da fibromialgia tanto em homens quanto em mulheres (Macfarlane et al., 2017).

De acordo com a World Health Organization, as doenças musculoesqueléticas acarretam grande despesas com a saúde e influenciam em perdas relacionadas ao trabalho (World Health Organization, 2020). Sabe-se, também, que o treinamento resistido, quando realizado em intensidade leve à moderada, pode proporcionar melhorias na qualidade do sono, prevenir a sarcopenia e diminuir dores e fadiga muscular (Souza \& Amorim, 2016). Além disso, evidências sugerem que o treinamento resistido apresenta efeitos positivos sobre os sintomas da fibromialgia, melhorando a força muscular, o sono, a capacidade funcional e reduzindo a intensidade da dor (Andrade, Vilarino, \& Bevilacqua, 2017; Andrade et al., 2020). O treinamento resistido, portanto, poderia auxiliar no tratamento de efeitos negativos da fibromialgia, minimizando o ônus que este quadro clínico apresenta aos diferentes setores de saúde.

A partir de um levantamento realizado na literatura, contatou-se que pouco se sabe sobre os efeitos do exercício físico sobre a saúde de pessoas que possuem algum tipo de síndrome reumática. Além disso, a fibromialgia é uma condição que acomete predominantemente o sexo feminino (Martinez et al., 2017; Souza \& Perissinotti, 2018), o que denota a necessidade de realizar investigações que envolvam este grupo específico. Diante do exposto, o objetivo desta revisão foi analisar estudos 
que avaliaram a influência do treinamento resistido sobre a saúde de mulheres com fibromialgia.

\section{Metodologia}

O presente estudo trata-se de uma revisão integrativa, método que possibilita a inclusão de estudos com diferentes delineamentos, com o intuito de compreender o problema analisado (Souza, Silva, \& Carvalho, 2010). Foram incluídos nesta revisão estudos publicados entre janeiro de 2016 e novembro de 2020, que analisaram o efeito do treinamento resistido sobre a saúde de mulheres adultas com fibromialgia. Estudos de revisão, teses, dissertações e relatórios governamentais não foram considerados nesta análise. Foram excluídos desta revisão estudos que analisaram mulheres com idade inferior a 18 anos ou superior a 64 anos.

A busca e seleção dos estudos foi realizada por dois autores (FJB e JRML), de forma individual. Quando não houve consenso durante a seleção dos estudos, um terceiro revisor foi consultado (BFC). A estratégia de busca adotada foi: fibromyalgia OR fibromyalgia syndrome AND women AND adults AND resistance training. As bases de dados utilizadas foram Pubmed, SciELO e Biblioteca Virtual em Saúde (BVS). As informações extraídas a partir dos estudos incluídos na revisão foram autor, ano de publicação, país, número de participantes, desenho do estudo, determinação da fibromialgia (autorrelato ou diagnóstico clínico), tempo de intervenção (se houver), protocolo utilizado na intervenção (tipo de exercício, frequência, intensidade, número de repetições e intervalo de descanso) e resultados.

\section{Resultados}

A Figura 1 apresenta o fluxograma do processo de seleção dos estudos. Inicialmente, foram identificados 1.368 artigos nas bases de dados investigadas. Após a aplicação do filtro (artigos publicados nos últimos 5 anos), 838 estudos apresentaram relevância potencial, sendo que 604 estavam indexados no Pubmed e 234 na BVS. Destes, 813 artigos foram excluídos após leitura de títulos e resumos. O restante dos estudos $(n=25)$ foram selecionados para a leitura na íntegra e importados para um software gerenciador de referências (EndNote Web). Desse total, 14 artigos foram excluídos com justificativa e 11 atenderam aos critérios de inclusão.

As características dos estudos incluídos nesta revisão $(n=11)$ foram apresentadas na Tabela 1 . $\mathrm{O}$ ano de publicação dos estudos incluídos variou entre 2016 e 2020. A maioria dos estudos foi desenvolvida na Suécia (Bjersing et al., 2017; Ericsson et al., 2016; Ernberg et al., 2016; Gerdle et al., 2016; Larsson et al., 2017; Palstam et al., 2016) e no Brasil (Andrade, Azevedo Klumb Steffens, Mendes Sieczkowska, Reis Coimbra, \& Torres Vilarino, 2019a; Assumpção et al., 2018; Silva et al., 2019). A amostra variou entre 28 e 133 participantes, totalizando em 820 mulheres envolvidas nesta revisão. O ensaio clínico de Andrade et al. (2019a) apresentou o menor tempo de intervenção (três sessões de treinamento), enquanto, na maioria dos estudos (Bjersing et al., 2017; Ericsson et al., 2016; Ernberg et al., 2016; Gerdle et al., 2016; Larsson et al., 2017; Palstam et al., 2016), o tempo de intervenção foi de 15 semanas. A fibromialgia foi determinada por meio de diagnóstico clínico em todos os trabalhos incluídos na revisão. 
Figura 1 - Fluxograma de identificação e seleção dos estudos.

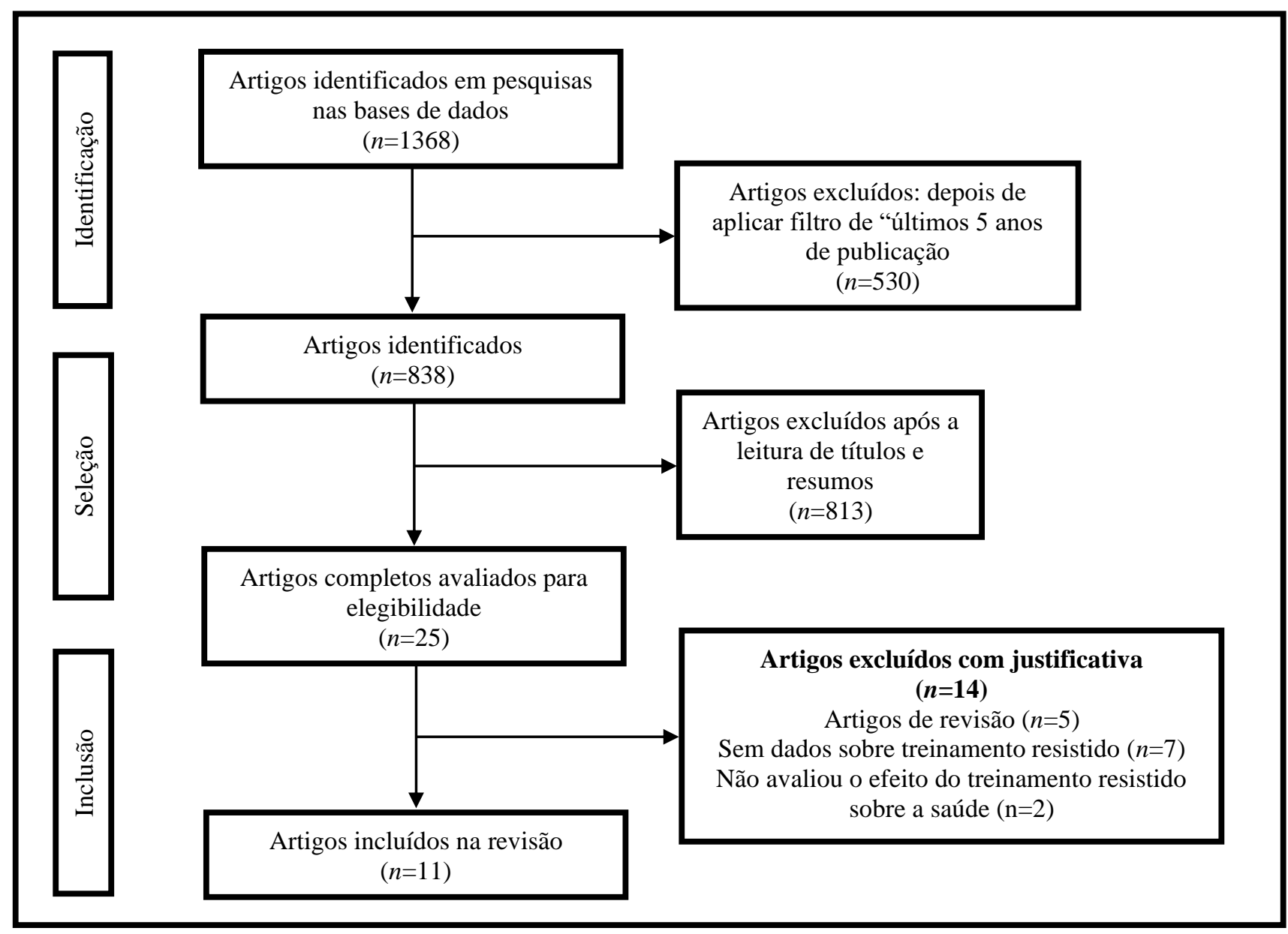

Fonte: Autores.

\section{Discussão}

A partir desta revisão, evidenciou-se que a intervenção realizada por meio do treinamento resistido foi benéfica para a saúde de mulheres adultas com fibromialgia no que se refere à saúde física, mental e qualidade de vida. Deste modo, o treinamento resistido contribuiu para a redução da dor e da fadiga, redução do escore de depressão, com a melhora do condicionamento físico, com o aumento da força e da resistência, com a melhora do humor e da qualidade de vida nos domínios relacionados aos aspectos físicos, sociais, emocionais, mentais e à vitalidade.

Corroborando com os achados da presente investigação, um estudo de revisão sistemática constatou que o treinamento resistido realizado de forma contínua e orientada, a partir de sessões com duração mínima de 30 minutos, de duas a três vezes por semana, diminuiu a dor em mulheres com fibromialgia, sendo que melhoras significativas foram observadas a partir da oitava semana de treinamento (Correia, Lima Filho, Fontes, Varella, \& Brasileiro, 2018). A partir do Consenso Brasileiro de Fibromialgia, dentre os tratamentos não medicamentosos recomendados para a fibromialgia, destaca-se a realização de exercícios musculoesqueléticos por, pelo menos, duas vezes por semana, considerando a progressão lenta e gradual desses exercícios (Heymann et al., 2010). Apesar dos estudos incluídos nesta revisão terem utilizado diferentes protocolos de treinamento, percebe-se que todos realizaram a intervenção com a frequência mínima de duas vezes por semana e, por isso, estão em consonância com as recomendações atuais. 
Tabela 1 - Características dos estudos incluídos na revisão.

\begin{tabular}{|c|c|c|c|c|c|c|}
\hline Autores (ano) & País & $\begin{array}{c}\mathrm{N}^{\mathbf{0}} \text { de } \\
\text { participantes }\end{array}$ & $\begin{array}{l}\text { Desenho do } \\
\text { estudo }\end{array}$ & $\begin{array}{l}\text { Duração da } \\
\text { intervenção }\end{array}$ & Protocolo de treinamento & Resultados \\
\hline $\begin{array}{l}\text { Andrade et al. } \\
\qquad \text { (2019a) }\end{array}$ & Brasil & 28 & Ensaio clínico & 3 sessões & $\begin{array}{c}\text { Frequência: } 3 \text { vezes/semana } \\
\text { Intervalo entre sessões: } 48 \text { horas } \\
\text { Duração da sessão: } 60 \text { min. } \\
\text { Treinamento: aquecimento (10 min.) seguido de } 50 \\
\text { min. de TR ( } 3 \text { séries de } 12 \text { repetições com descanso } \\
\text { entre séries de } 1 \text { min.) } \\
\end{array}$ & $\begin{array}{l}\text { Uma única sessão de treino de força foi } \\
\text { suficiente para melhorar o estado de humor } \\
\text { de pacientes com fibromialgia. O resultado } \\
\text { da terceira sessão foi semelhante ao obtido } \\
\text { na primeira sessão. }\end{array}$ \\
\hline $\begin{array}{l}\text { Assumpção et } \\
\text { al. (2018) }\end{array}$ & Brasil & 44 & $\begin{array}{c}\text { Ensaio clínico } \\
\text { randomizado }\end{array}$ & 12 semanas & $\begin{array}{c}\text { Frequência: } 2 \text { vezes/semana } \\
\text { Duração da sessão: } 40 \text { min. } \\
\text { Treinamento: sobrecarga progressiva } \\
\text { (1 série de } 8 \text { repetições) }\end{array}$ & $\begin{array}{l}\text { Melhoria no limiar de dor e na QV na } \\
\text { função física, social, emocional, vitalidade e } \\
\text { saúde mental (menor escore de depressão). }\end{array}$ \\
\hline $\begin{array}{l}\text { Bjersing et al. } \\
\quad \text { (2017) }\end{array}$ & Suécia & 43 & $\begin{array}{l}\text { Ensaio clínico } \\
\text { randomizado } \\
\text { controlado }\end{array}$ & 15 semanas & $\begin{array}{c}\text { Frequência: } 2 \text { vezes/semana } \\
\text { Treinamento: aquecimento (10 min.) seguido de } 50 \\
\text { minutos TR } \\
\text { Descanso entre séries: } 1 \text { minuto }\end{array}$ & $\begin{array}{l}\text { Mulheres magras apresentaram melhora em } \\
\text { relação à dor atual, fadiga geral e força de } \\
\text { flexão do cotovelo quando comparadas às } \\
\text { mulheres com sobrepeso ou obesas. }\end{array}$ \\
\hline $\begin{array}{l}\text { Ericsson et al. } \\
\qquad(2016)\end{array}$ & Suécia & 130 & $\begin{array}{l}\text { Ensaio clínico } \\
\text { randomizado } \\
\text { controlado }\end{array}$ & 15 semanas & $\begin{array}{c}\text { Frequência: } 2 \text { vezes/semana } \\
\text { Treinamento: aquecimento (10 min.) seguido de } 50 \\
\text { min. de TR } \\
\text { Descanso entre séries: } 1 \text { min. }\end{array}$ & $\begin{array}{l}\text { O TR contribuiu para a melhora da fadiga } \\
\text { física em mulheres com fibromialgia quando } \\
\text { comparadas ao grupo de controle ativo. }\end{array}$ \\
\hline $\begin{array}{l}\text { Ernberg et al. } \\
\text { (2016) }\end{array}$ & Suécia & 51 & $\begin{array}{l}\text { Ensaio clínico } \\
\text { randomizado } \\
\text { controlado }\end{array}$ & 15 semanas & $\begin{array}{l}\text { Frequência: } 2 \text { vezes/semana } \\
\text { Treinamento: aquecimento (10 min.) seguido de } 50 \\
\text { min. de TR com sobrecarga progressiva } \\
\text { Descanso entre séries: } 1 \text { min. }\end{array}$ & $\begin{array}{c}\text { Apesar do TR ter melhorado a força } \\
\text { muscular do braço e reduzido a dor geral, } \\
\text { não houveram alterações nos níveis de dor, } \\
\text { fadiga ou citocinas ( IL-1 } \beta \text {, IL-6, IL-8 ou } \\
\text { TNF). }\end{array}$ \\
\hline $\begin{array}{l}\text { Gerdle et al. } \\
\quad(2016)\end{array}$ & Suécia & 133 & $\begin{array}{l}\text { Ensaio clínico } \\
\text { randomizado } \\
\text { controlado }\end{array}$ & 15 semanas & $\begin{array}{c}\text { Frequência: } 2 \text { vezes/semana } \\
\text { Treinamento: aquecimento (10 min.) seguido de } 50 \\
\text { min. de TR com progressão de carga } \\
\text { Descanso entre séries: } 1 \text { min. }\end{array}$ & $\begin{array}{c}\text { Após intervenção, o grupo que realizou o TR } \\
\text { aumentou a força e resistência e apresentou } \\
\text { redução na intensidade da dor e nas } \\
\text { concentrações intersticiais de glutamato, } \\
\text { piruvato e lactato. }\end{array}$ \\
\hline
\end{tabular}


Research, Society and Development, v. 10, n. 5, e29410514674, 2021

(CC BY 4.0) | ISSN 2525-3409 | DOI: http://dx.doi.org/10.33448/rsd-v10i5.14674

\begin{tabular}{|c|c|c|c|c|c|c|}
\hline $\begin{array}{l}\text { Glasgow, Stone, } \\
\text { and Kingsley } \\
\text { (2017) }\end{array}$ & EUA & 35 & $\begin{array}{l}\text { Ensaio clínico } \\
\text { randomizado }\end{array}$ & 8 semanas & $\begin{array}{c}\text { Frequência: } 2 \text { vezes/semana } \\
\text { Duração da sessão: } 30 \text { min. } \\
\text { Descanso entre séries: } 90 \text { segundos } \\
\text { Treinamento: } 3 \text { séries de } 8 \text { a } 12 \text { repetições }\end{array}$ & $\begin{array}{l}\text { Após } 8 \text { semanas de intervenção com TR } \\
\text { observou-se o aumento da força máxima e } \\
\text { redução do impacto da fibromialgia, } \\
\text { proporcionando melhor QV. }\end{array}$ \\
\hline $\begin{array}{l}\text { Izquierdo- } \\
\text { Alventosa et al. } \\
\quad(2020)\end{array}$ & Espanha & 32 & $\begin{array}{c}\text { Ensaio clínico } \\
\text { randomizado }\end{array}$ & 8 semanas & $\begin{array}{c}\text { Duração da intervenção: } 8 \text { semanas } \\
\text { Frequência: } 2 \text { vezes/semana } \\
\text { Duração da sessão: } 60 \text { min. } \\
\text { Treinamento: Cada sessão de treinamento foi } \\
\text { dividida em } 3 \text { partes (aquecimento, treinamento e } \\
\text { desaquecimento). No TR houve aumento } \\
\text { progressivo de carga. }\end{array}$ & $\begin{array}{l}\text { Um programa combinado de exercícios } \\
\text { físicos de baixa intensidade incluindo TR e } \\
\text { de coordenação melhorou aspectos } \\
\text { psicológicos (ansiedade e estresse), limiar de } \\
\text { dor, QV e condicionamento físico em } \\
\text { mulheres com fibromialgia. }\end{array}$ \\
\hline $\begin{array}{l}\text { Larsson et al. } \\
\qquad(2017)\end{array}$ & Suécia & 67 & $\begin{array}{l}\text { Ensaio clínico } \\
\text { randomizado } \\
\text { controlado }\end{array}$ & 15 semanas & $\begin{array}{l}\text { Frequência: } 2 \text { vezes/semana } \\
\text { Treinamento: aquecimento (10 min.) seguido de } 50 \\
\text { min. de TR } \\
\text { Descanso entre séries: } 1 \text { min. }\end{array}$ & $\begin{array}{l}\text { Melhora da força muscular, redução na } \\
\text { intensidade da dor e aumento na quantidade } \\
\text { de AF após a intervenção. }\end{array}$ \\
\hline $\begin{array}{l}\text { Palstam et al. } \\
\qquad(2016)\end{array}$ & Suécia & 67 & $\begin{array}{l}\text { Ensaio clínico } \\
\text { randomizado } \\
\text { controlado }\end{array}$ & 15 semanas & $\begin{array}{c}\text { Frequência: } 2 \text { vezes/semana } \\
\text { Treinamento: aquecimento (10 min.) seguido de } 50 \\
\text { min. de TR } \\
\text { Descanso entre séries: } 1 \text { min. }\end{array}$ & $\begin{array}{c}\text { O TR possibilitou a melhora da força } \\
\text { muscular, redução na intensidade da dor e } \\
\text { aumento na quantidade de AF após a } \\
\text { intervenção. Além disso, a intervenção } \\
\text { baseada em princípios de auto eficácia teve } \\
\text { um efeito positivo na deficiência recreativa, } \\
\text { social e ocupacional. }\end{array}$ \\
\hline $\begin{array}{l}\text { Silva et al. } \\
\text { (2019) }\end{array}$ & Brasil & 60 & $\begin{array}{l}\text { Ensaio clínico } \\
\text { randomizado } \\
\text { controlado }\end{array}$ & 12 semanas & $\begin{array}{c}\text { Frequência: } 2 \text { vezes/semana } \\
\text { Duração da sessão: } 40 \text { min. } \\
\text { Descanso entre séries: } 1 \text { a } 2 \text { min. }\end{array}$ & $\begin{array}{c}\text { Houve melhora na força e capacidade } \\
\text { funcional no grupo de mulheres que realizou } \\
\text { o TR quando comparas àquelas que } \\
\text { participaram do grupo sofrologia } \\
\text { (relaxamento). }\end{array}$ \\
\hline
\end{tabular}

QV: Qualidade de vida; TR: Treinamento resistido; min.: minutos. Fonte: Autores, com base nos dados da pesquisa 
Um estudo envolvendo 284 indivíduos com fibromialgia (97,9\% mulheres) evidenciou que aqueles que se exercitavam regularmente apresentavam melhor qualidade de vida geral quando comparados aos que não se exercitavam de forma frequente (Sieczkowska, Vilarino, de Souza, \& Andrade, 2020), indo ao encontro dos resultados desta revisão. A adoção de um estilo de vida mais ativo pode contribuir para a melhoria da saúde de seus praticantes (Camilo, 2019). Desta forma, indivíduos que se exercitam de forma regular podem obter efeitos benéficos que impactem diretamente na qualidade de vida, ao longo dos anos.

Os efeitos positivos do treinamento resistido sobre a saúde física e mental de pessoas com fibromialgia têm sido difundidos na literatura. A partir de uma revisão sistemática que analisou 12 estudos, evidenciou-se que o treinamento resistido se associou à redução dos sintomas de ansiedade e depressão, bem como ao aumento da força muscular (Andrade et al., 2019b). De forma semelhante, um estudo de intervenção que realizou um programa de exercícios resistidos demonstrou, após 15 semanas, o aumento da força muscular e dos níveis de anandamida, além da redução da dor e dos sintomas depressivos (Stensson et al., 2020). A anandamida é um neurotransmissor endógeno capaz de promover maior sensação de felicidade. Além disso, a produção de outros neurotransmissores, como serotonina, dopamina e endorfina, responsáveis pelo aumento da sensação de prazer, bem-estar e melhora da qualidade do sono pode ser estimulada a partir da realização de exercícios físicos (Gupta, Goyal, Saraf, \& Thareja, 2019). Dessa maneira, o treinamento resistido pode contribuir para o aumento da produção de hormônios responsáveis pela melhora do humor, o que poderia contribuir para a redução de problemas relacionados à depressão e, consequentemente, oportunizaria uma melhora da qualidade de vida.

A partir desta investigação, constatou-se que a maior parte dos estudos incluídos ( $\mathrm{n}=6$ ) foi desenvolvida na Suécia e constituiu-se de subamostras de um mesmo grupo, o que denota a escassez de investigações relacionadas à temática no período analisado. Somado a isso, a carência de estudos nacionais dificulta a generalização destes achados para a população brasileira e dificulta a realização deste tipo de pesquisa, uma vez que impossibilita a definição de um protocolo específico baseado no tipo de exercício físico, frequência, duração, intensidade e tempo de intervalo, capaz de atenuar os efeitos adversos da fibromial gia sobre a saúde.

\section{Conclusão}

A partir deste estudo de revisão, foi possível constatar que o treinamento resistido é um importante método não medicamentoso capaz de atenuar os efeitos da fibromialgia em mulheres adultas, contribuindo para a melhoria da qualidade de vida e da saúde física e mental. Especificamente, o treinamento resistido apresentou efeitos benéficos no que se refere à redução da dor e da fadiga, redução no escore de depressão, melhora do condicionamento físico, aumento da força e da resistência e melhora do humor e da qualidade de vida.

Os achados apresentados evidenciam a necessidade de rever as políticas públicas de saúde existentes, a fim de estimular a associação do treinamento resistido com outros métodos de tratamento convencional para mulheres adultas com problemas reumáticos, de forma a atenuar os efeitos da fibromialgia e reduzir o ônus desse problema ao sistema de saúde. Destaca-se, ainda, que apenas três estudos brasileiros foram identificados nesta revisão, no recorte de tempo investigado. Por essa razão, sugere-se a realização de novas pesquisas nacionais, que utilizem diferentes protocolos de treinamento a fim de ampliar o conhecimento acerca da temática em questão.

\section{Referências}

Andrade, A., Azevedo Klumb Steffens, R., Mendes Sieczkowska, S., Reis Coimbra, D., \& Torres Vilarino, G. (2019a). Acute effect of strength training on mood of patients with fibromyalgia syndrome. Reumatismo, 71(3), 141-147. 10.4081/reumatismo.2019.1169

Andrade, A., de Azevedo Klumb Steffens, R., Peyré Tartaruga, L. A., Sieczkowska, S. M., \& Torres Vilarino, G. (2018). A systematic review of the effects of strength training in patients with fibromyalgia: clinical outcomes and design considerations. Adv Rheumatol, 58(1), 36. 10.1186/s42358-018-0033-9 
Andrade, A., Vilarino, G. T., \& Bevilacqua, G. G. (2017). What Is the Effect of Strength Training on Pain and Sleep in Patients With Fibromyalgia? Am J Phys Med Rehabil, 96(12), 889-893. 10.1097/phm.0000000000000782

Andrade, A., Vilarino, G. T., de Souza, C. A., Sieczkowska, S. M., Serafim, T. T., \& Pereira Júnior, A. A. (2019b). Modulation of Autonomic Function by Physical Exercise in Patients with Fibromyalgia Syndrome: A Systematic Review. PM R., 11(10), 1121-1131. 10.1002/pmrj.12158

Andrade, A., Vilarino, G. T., Sieczkowska, S. M., Coimbra, D. R., Bevilacqua, G. G., \& Steffens, R. A. K. (2020). The relationship between sleep quality and fibromyalgia symptoms. J Health Psychol, 25(9), 1176-1186. 10.1177/1359105317751615

Assumpção, A., Matsutani, L. A., Yuan, S. L., Santo, A. S., Sauer, J., Mango, P., \& Marques, A. P. (2018). Muscle stretching exercises and resistance training in fibromyalgia: which is better? A three-arm randomized controlled trial. Eur J Phys Rehabil Med, 54(5), 663-670. 10.23736/s1973-9087.17.04876-6

Bidonde, J., Busch, A. J., Schachter, C. L., Overend, T. J., Kim, S. Y., Góes, S. M., Boden, C., \& Foulds, H. J. (2017). Aerobic exercise training for adults with fibromyalgia. The Cochrane database of systematic reviews, 6(6), CD012700-CD012700. 10.1002/14651858.CD012700

Bjersing, J. L., Larsson, A., Palstam, A., Ernberg, M., Bileviciute-Ljungar, I., Löfgren, M., Gerdle, B., Kosek, E., \& Mannerkorpi, K. (2017). Benefits of resistance exercise in lean women with fibromyalgia: involvement of IGF-1 and leptin. BMC Musculoskelet Disord, 18(1), 106. 10.1186/s12891-017-1477-5

Camilo, B. F. (2019). Sentar-se menos e mover-se mais: o papel da atividade física na prevenção da obesidade. Arq Cien Esp, 7(3), 100.

Clauw, D. J. (2014). Fibromyalgia: a clinical review. Jama, 311(15), 1547-1555. 10.1001/jama.2014.3266

Correia, L. C., Lima Filho, B. F., Fontes, F. P., Varella, L. R. D., \& Brasileiro, J. S. (2018). Efeito do treinamento resistido na redução da dor no tratamento de mulheres com fibromialgia: revisão sistemática. Rev Bras Ciênc Mov, 26(2), 170-175.

Ericsson, A., Palstam, A., Larsson, A., Löfgren, M., Bileviciute-Ljungar, I., Bjersing, J., Gerdle, B., Kosek, E., \& Mannerkorpi, K. (2016). Resistance exercise improves physical fatigue in women with fibromyalgia: a randomized controlled trial. Arthritis Res Ther, 18, 176. 10.1186/s13075-016-1073-3

Ernberg, M., Christidis, N., Ghafouri, B., Bileviciute-Ljungar, I., Löfgren, M., Larsson, A., Palstam, A., Bjersing, J., Mannerkorpi, K., Kosek, E., \& Gerdle, B. (2016). Effects of 15 weeks of resistance exercise on pro-inflammatory cytokine levels in the vastus lateralis muscle of patients with fibromyalgia. Arthritis Res Ther, 18(1), 137. 10.1186/s13075-016-1041-y

Gerdle, B., Ernberg, M., Mannerkorpi, K., Larsson, B., Kosek, E., Christidis, N., \& Ghafouri, B. (2016). Increased Interstitial Concentrations of Glutamate and Pyruvate in Vastus Lateralis of Women with Fibromyalgia Syndrome Are Normalized after an Exercise Intervention - A Case-Control Study. PLoS One, 11(10), e0162010. 10.1371/journal.pone.0162010

Glasgow, A., Stone, T. M., \& Kingsley, J. D. (2017). Resistance Exercise Training on Disease Impact, Pain Catastrophizing and Autonomic Modulation in Women with Fibromyalgia. Int J Exerc Sci, 10(8), 1184-1195.

Gupta, J. K., Goyal, A., Saraf, B., \& Thareja, K. (2019). Scientific Basis for Deplorable Women's Behaviour: A Connection with Hormonal Imbalance. Indian J Sci Technol, 12(16), 1-7. 10.17485/ijst/2019/v12i16/143844

Häuser, W., Ablin, J., Fitzcharles, M.-A., Littlejohn, G., Luciano, J. V., Usui, C., \& Walitt, B. (2015). Fibromyalgia. Nat Rev Dis Primers, 1(1), 15022. 10.1038/nrdp.2015.22

Heymann, R. E., Paiva, E. d. S., Helfenstein Junior, M., Pollak, D. F., Martinez, J. E., Provenza, J. R., Paula, A. P., Althoff, A. C., Souza, E. J. d. R., Neubarth, F., Lage, L. V., Rezende, M. C., Assis, M. R., Lopes, M. L. L., Jennings, F., Araújo, R. L. C. d. C., Cristo, V. V., Costa, E. D. G., Kaziyama, H. H. S., Yeng, L. T., Iamamura, M., Saron, T. R. P., Nascimento, O. J. M., Kimura, L. K., Leite, V. M., Oliveira, J., Araújo, G. T. B., \& Fonseca, M. C. M. (2010). Consenso brasileiro do tratamento da fibromialgia. Rev Bras Reumatol, 50(1), 56-66. 10.1590/S0482-50042010000100006

Izquierdo-Alventosa, R., Inglés, M., Cortés-Amador, S., Gimeno-Mallench, L., Chirivella-Garrido, J., Kropotov, J., \& Serra-Añó, P. (2020). Low-Intensity Physical Exercise Improves Pain Catastrophizing and Other Psychological and Physical Aspects in Women with Fibromyalgia: A Randomized Controlled Trial. Int J Environ Res Public Health, 17(10). 10.3390/ijerph17103634

Jorge, L. L., Tomikawa, L. C. O., \& Jucá, S. S. (2007). Efeito de um programa de reabilitação multidisciplinar para homens portadores de fibromialgia: estudo aleatorizado controlado. Acta fisiátrica, 14(4), 196-203.

Larsson, A., Palstam, A., Löfgren, M., Ernberg, M., Bjersing, J., Bileviciute-Ljungar, I., Gerdle, B., Kosek, E., \& Mannerkorpi, K. (2017). Pain and fear avoidance partially mediate change in muscle strength during resistance exercise in women with fibromyalgia. J Rehabil Med, 49(9), 744-750. $10.2340 / 16501977-2278$

Lima, H. F., Badaró, R. R., \& Ladeia, A. P. A. (2019). A importância do exercício físico em pacientes fibromiálgicos. In H. N. P. Oliva (Ed.), Anais do II Congresso Norte-Mineiro Multidisciplinar das Patologias da Coluna Vertebral (II Medular) e I Congresso Norte-Mineiro de Dor (Vol. 1, pp. 19-20). Montes Claros.

Macfarlane, G. J., Kronisch, C., Dean, L. E., Atzeni, F., Häuser, W., Fluß, E., Choy, E., Kosek, E., Amris, K., Branco, J., Dincer, F., Leino-Arjas, P., Longley, K., McCarthy, G. M., Makri, S., Perrot, S., Sarzi-Puttini, P., Taylor, A., \& Jones, G. T. (2017). EULAR revised recommendations for the management of fibromyalgia. Ann Rheum Dis, 76(2), 318-328. 10.1136/annrheumdis-2016-209724

Martinez, J. E., Paiva, E. S., Rezende, M. C., Heymann, R. E., Helfenstein Jr, M., Ranzolin, A., Provenza, J. R., Ribeiro, L. S., Souza, E. J., \& Feldman, D. P. (2017). EpiFibro (Registro Brasileiro de Fibromialgia): dados sobre a classificação do ACR e preenchimento dos critérios diagnósticos preliminares e avaliação de seguimento. Rev Bras Reumatol, 57(2), 129-133. 10.1016/j.rbre.2016.09.012

Okifuji, A., \& Turk, D. C. (2015). Behavioral and Cognitive-Behavioral Approaches to Treating Patients with Chronic Pain: Thinking Outside the Pill Box. J Rat-Emo Cognitive-Behav Ther, 33(3), 218-238. 10.1007/s10942-015-0215-x 
Research, Society and Development, v. 10, n. 5, e29410514674, 2021

(CC BY 4.0) | ISSN 2525-3409 | DOI: http://dx.doi.org/10.33448/rsd-v10i5.14674

Palstam, A., Larsson, A., Löfgren, M., Ernberg, M., Bjersing, J., Bileviciute-Ljungar, I., Gerdle, B., Kosek, E., \& Mannerkorpi, K. (2016). Decrease of fear avoidance beliefs following person-centered progressive resistance exercise contributes to reduced pain disability in women with fibromyalgia: secondary exploratory analyses from a randomized controlled trial. Arthritis Res Ther, 18(1), 116. 10.1186/s13075-016-1007-0

Sieczkowska, S. M., Vilarino, G. T., de Souza, L. C., \& Andrade, A. (2020). Does physical exercise improve quality of life in patients with fibromyalgia? Ir J Med Sci, 189(1), 341-347. 10.1007/s11845-019-02038-z

Silva, H. J. A., Assunção Júnior, J. C., de Oliveira, F. S., Oliveira, J. M. P., Figueiredo Dantas, G. A., Lins, C. A. A., \& de Souza, M. C. (2019). Sophrology versus resistance training for treatment of women with fibromyalgia: A randomized controlled trial. J Bodyw Mov Ther, 23(2), 382-389. 10.1016/j.jbmt.2018.02.005

Sluka, K. A., \& Clauw, D. J. (2016). Neurobiology of fibromyalgia and chronic widespread pain. Neuroscience, 338, 114-129. 10.1016/j.neuroscience.2016.06.006

Souza, E., \& Amorim, L. M. (2016). Benefícios dos exercícios resistidos em pacientes portadores de fibromialgia: revisão bibliográfica. Amazônia: Science \& Health, 4(1), 30-34

Souza, J. B., \& Perissinotti, D. M. N. (2018). The prevalence of fibromyalgia in Brazil-a population-based study with secondary data of the study on chronic pain prevalence in Brazil. BrJP, 1(4), 345-348. 10.5935/2595-0118.20180065

Souza, M. T. d., Silva, M. D. d., \& Carvalho, R. d. (2010). Integrative review: what is it? How to do it? Einstein, 8(1), 102-106. 10.1590/s167945082010rw1134

Stensson, N., Gerdle, B., Ernberg, M., Mannerkorpi, K., Kosek, E., \& Ghafouri, B. (2020). Increased Anandamide and Decreased Pain and Depression after Exercise in Fibromyalgia. Med sci sports exerc, 52(7), 1617-1628. 10.1249/mss.0000000000002293

Wolfe, F., Clauw, D. J., Fitzcharles, M.-A., Goldenberg, D. L., Häuser, W., Katz, R. L., Mease, P. J., Russell, A. S., Russell, I. J., \& Walitt, B. (2016). 2016 Revisions to the 2010/2011 fibromyalgia diagnostic criteria. Seminars in Arthritis and Rheumatism, 46(3), 319-329. 10.1016/j.semarthrit.2016.08.012

World Health Organization. (2020). Chronic diseases and health promotion: Chronic rheumatic conditions. https://www.who.int/chp/topics/rheumatic/en/ 